\title{
Biaxial experiments and numerical analyses on damage prediction in metal forming processes
}

\author{
Michael Brünig ${ }^{1, a}$, Steffen Gerke ${ }^{1}$, and Marco Schmidt ${ }^{1}$ \\ ${ }^{1}$ Institut für Mechanik und Statik, Universität der Bundeswehr München, 85577 Neubiberg, Germany
}

\begin{abstract}
The paper discusses an anisotropic continuum damage and failure model for ductile metals. The phenomenological approach is based on kinematic definition of damage tensors and takes into account the effect of stress state on damage conditions and damage strain evolution laws. Different branches of these criteria based on experimental studies and numerical simulations are considered corresponding to various microscopic damage and fracture mechanisms depending on stress intensity, stress triaxiality and the Lode parameter. Experiments with biaxially loaded specimens and corresponding numerical simulations have been performed showing that they cover a wide range of stress states in combined tension, shear and compression regimes. Digital image correlation technique has been used to analyze current strain states in critical regions of the specimens. Damage behavior for shear-compression loading conditions appearing in metal forming processes is discussed in detail.
\end{abstract}

\section{Introduction}

Numerical calculations for optimization of forming processes currently receive remarkable attention because products of modern metal forming processes have to fulfill economic, environmental and material strength requirements caused by the increasing demands of the customers. Therefore, the use of high quality metals like high strength steels, advanced high strength steels and aluminum alloys has been increased during the last decades. There are, for example, requirements on lightweight design leading to improved energy consumption or cost efficiency and, at the same time, to enforce the safety requirements. These aspects demand enhancement of material properties to avoid early localization of inelastic strains as well as damage and fracture of structural components under complex loading conditions. Hence, numerical simulation of various metal forming processes becomes a indispensable practical tool in modern technologies. This requires highly predictive and practically applicable constitutive theories as well as corresponding efficient numerical methods. Therefore, accurate modeling and numerical simulation of large inelastic deformations as well as of damage and failure behavior in materials and structures are of keen interest for many metal forming processes. In various experiments and forming operations with ductile metals large and often localized inelastic deformations occur which may be accompanied by different damage and fracture mechanisms on the micro- and meso-scales. Formation of damage on the micro-level may then lead to macro-cracks and to final failure of structural elements. Since industrial processes like rolling and forging often show remarkable negative

\footnotetext{
${ }^{\text {a } C o r r e s p o n d i n g ~ a u t h o r: ~ m i c h a e l . b r u e n i g @ u n i b w . d e ~}$
}

hydrostatic stress states damage and fracture mechanisms at negative stress triaxialities are here of special interest.

Characteristics of damage and failure mechanisms depend on stress state acting in a material point. For example during tension loading with high positive stress triaxialities damage in ductile metals is mainly caused by nucleation, growth and coalescence of micro-voids whereas during shear and compression loading with nearly zero or negative stress triaxialities the main damage mechanisms on the micro-scale are formation, growth and coalescence of micro-shear-cracks. Combination of these basic microscopic damage mechanisms occurs for moderate positive stress triaxialities whereas no damage has been observed in ductile metals for high negative stress triaxialities. Thus, ductile damage behavior and crack formation strongly depend on the stress triaxiality. Hence, development of accurate and realistic phenomenological continuum models must be based on detailed experimental and numerical analyses on both the macro- and the microlevel to investigate and to understand these complex stressstate-dependent damage and fracture processes. Corresponding constitutive equations have to be evaluated and quantification of damage amount at various stress states needs to be performed to be able to predict and to judge quality of metal forming processes.

Combination of investigations on both the micro- and the macro-level has widely enriched the understanding of damage and fracture processes in metals. In particular, from microscopic point of view ductile fracture in metals is the integral process of nucleation, growth and coalescence of the different micro-defects discussed above. For example, detailed information on stress state dependence of these damage and fracture processes on the micro-level 
can be obtained by numerical simulations considering representative volume elements under various loading conditions [1-5]. These microscopic numerical calculations allow detailed analysis of individual behavior of microdefects and it was possible to detect a number of damage and failure mechanisms which have not been revealed by experiments alone. Interpretation of numerical results of these calculations on the micro-scale allow proposition of evolution equations for chosen damage parameters and of equations for damage and fracture criteria as well as of evolution laws for damage strain rate tensors depending on the stress state $[4,5]$. Since the proposed criteria and evolution equations are only based on numerical analyses experimental validation is still necessary.

Experiments with carefully designed metal specimens have been proposed and discussed in the literature. In particular, uniaxial tension tests with un-notched and notched specimens and corresponding numerical calculations have been performed to study the dependence of damage and failure on different positive stress triaxialities [6-9]. In addition, new geometries of uniaxially loaded specimens have been developed to investigate the behavior under nearly zero stress triaxialities where shear mechanisms occur in the critical parts of the specimens [6, 7, 9, 10]. Furthermore, to examine the behavior under wider regions of stress triaxialities butterfly specimens with complex geometries have been developed which can be loaded in uniaxial tension tests in different directions using special experimental equipment $[8,11]$. Alternatively, twodimensional experiments and different geometries of cruciform specimens have been investigated [12]. In addition, biaxial experiments with new shear-tension-specimens in combination with numerical simulations have been proposed by $[13,14]$ to study stress-state-dependent damage and failure processes. Scanning electron microscope analyses of fracture surfaces elucidate different mechanisms of ductile fracture on the micro-level.

From theoretical point of view, anisotropic damage models taking into account tensorial damage variables are more suitable to simulate stress-state-dependent behavior but their practical applicability is often limited by large number of material parameters and difficulties in their identification. Therefore, in the present paper a phenomenological continuum damage approach based on simplified damage mode functions will be discussed. Experiments with bi-axially loaded specimens will be presented where evolution of strain fields are analyzed by digital image correlation in critical regions of the specimens where damage and failure occur. Numerical simulations based on the proposed continuum model have been performed and numerical results will be used to explain stress-state-dependent damage and failure processes especially in the regime of negative stress triaxialities.

\section{Continuum damage model}

The anisotropic continuum damage model proposed by [15] is used to predict the inelastic deformation behavior as well as the evolution of ductile damage and failure in ductile metals. This phenomenological approach applicable for different loading conditions is based on introduction of micro-structurally based damage variables that not only represent the volume fraction of the micro-defects but also take into account their current shape and orientation. In this context, in the proposed continuum damage model the damage process is governed by the evolution of damage strains on the macro-level caused by various stress-statedependent microscopic mechanisms of the micro-defects. Thus, the thermodynamically consistent continuum framework is based on kinematic description of damage leading to definition of damage strain rate tensors. The corresponding kinematic approach takes into account additive decomposition of the strain rate tensor into elastic, $\dot{\mathbf{H}}^{e l}$, effective plastic, $\dot{\overline{\mathbf{H}}}^{p l}$, and damage parts, $\dot{\mathbf{H}}^{d a}$. Furthermore, free energy functions with respect to undamaged and damaged configurations are introduced leading to elastic laws which in the damaged configuration are influenced by increasing damage to model the deteriorating effect of damage on elastic material properties, see [15] for further details. Considering the undamaged configurations plastic behavior is governed by a yield condition and a flow rule and, in a similar way, considering the damaged configurations damage behavior is characterized by a damage condition and a damage rule both depending on the stress triaxiality and the Lode parameter.

Equations characterizing elastic-plastic behavior of the undamaged matrix material are based on consideration of the effective undamaged configurations. The elastic behavior is described by an isotropic hyper-elastic law leading to the effective Kirchhoff stress tensor

$$
\overline{\mathbf{T}}=2 G \mathbf{A}^{e l}+\left(K-\frac{2}{3} G\right) \operatorname{tr}^{e l} \mathbf{1}
$$

where $G$ and $K$ are the constant shear and bulk modulus of the undamaged matrix material, respectively, and $\mathbf{A}^{e l}$ represents the elastic part of the strain tensor. In addition, plastic behavior is governed by the yield condition

$$
f^{p l}\left(\bar{I}_{1}, \bar{J}_{2}, c\right)=\sqrt{\bar{J}_{2}}-c\left(1-\frac{a}{c} \bar{I}_{1}\right)=0,
$$

with the first and second deviatoric invariant $\bar{I}_{1}=\operatorname{tr} \overline{\mathbf{T}}$ and $\bar{J}_{2}=\frac{1}{2} \operatorname{dev} \overline{\mathbf{T}} \cdot \operatorname{dev} \overline{\mathbf{T}}$ of the effective stress tensor (1), the strength coefficient $c$ of the matrix material and the hydrostatic stress coefficient $a$. In the present paper, the hydrostatic-stress-dependent isotropic yield function (2) is taken into account to adequately model the plastic behavior of metals. It has been shown in carefully performed experiments by [16] that even plastic yielding and hardening of metals shows small dependence on the hydrostatic stress. Numerical analyses discussed in [17] have shown that variation of the parameter $a$ in (2) remarkably affects the strain localization and failure behavior of specimens which is an important aspect in metal forming processes. On the other hand, metal sheets usually show anisotropic plastic behavior caused by the manufacturing process. However, in many metals the plastic anisotropy is small and, thus, the isotropic yield condition (2) seems to be an adequate approximation. For larger anisotropy 
the use of an anisotropic yield criterion as discussed, for example, in [18] is recommended.

Furthermore, plastic volumetric strains are marginal in metals [16]. Thus, the plastic potential function

$$
g^{p l}(\overline{\mathbf{T}})=\sqrt{\bar{J}_{2}}
$$

is introduced leading to the isochoric effective plastic strain rate

$$
\dot{\overline{\mathbf{H}}}^{p l}=\dot{\lambda} \frac{\partial g^{p l}}{\partial \overline{\mathbf{T}}}=\dot{\lambda} \frac{1}{2 \sqrt{\bar{J}_{2}}} \operatorname{dev} \overline{\mathbf{T}}=\dot{\gamma} \overline{\mathbf{N}}
$$

phenomenologically modeling the evolution of plastic deformations in ductile metals in an accurate manner. In Eq. (4) $\dot{\lambda}$ is a non-negative scalar-valued factor, $\overline{\mathbf{N}}=$ $\frac{1}{\sqrt{2 \bar{J}_{2}}} \operatorname{dev} \overline{\mathbf{T}}$ represents the normalized deviatoric stress tensor and $\dot{\gamma}=\overline{\mathbf{N}} \cdot \dot{\overline{\mathbf{H}}}^{p l}=\frac{1}{\sqrt{2}} \dot{\lambda}$ denotes the equivalent plastic strain rate measure used in the present constitutive model to characterize the amount of plastic deformation.

Moreover, the damaged configurations are considered to model the deformation behavior of anisotropically damaged ductile metals. It has been observed in many experiments that damage has remarkable influence on the elastic behavior of solids leading to material deterioration. Thus, the elastic constitutive law of the damaged material element takes into account both the elastic and the damage strain tensors, $\mathbf{A}^{e l}$ and $\mathbf{A}^{d a}$. Then, the Kirchhoff stress tensor with respect to the damaged configurations is given by

$$
\begin{aligned}
\mathbf{T}= & 2\left(G+\eta_{2} \operatorname{tr} \mathbf{A}^{d a}\right) \mathbf{A}^{e l} \\
& +\left(K-\frac{2}{3} G+2 \eta_{1} \operatorname{tr} \mathbf{A}^{d a}\right) \operatorname{tr} \mathbf{A}^{e l} \mathbf{1} \\
& +\eta_{3}\left(\mathbf{A}^{d a} \cdot \mathbf{A}^{e l}\right) \mathbf{1} \\
& +\eta_{3} \operatorname{tr} \mathbf{A}^{e l} \mathbf{A}^{d a} \\
& +\eta_{4}\left(\mathbf{A}^{e l} \mathbf{A}^{d a}+\mathbf{A}^{d a} \mathbf{A}^{e l}\right)
\end{aligned}
$$

where the additional constitutive parameters $\eta_{1}, \ldots, \eta_{4}$ are related to deteriorating effects of the macroscopic elastic material properties caused by different damage and failure mechanisms on the micro-level.

Determination of onset and continuation of damage is based on the concept of damage surface formulated in stress space $[15,19]$. Thus, the damage condition

$$
f^{d a}=\alpha I_{1}+\beta \sqrt{J_{2}}-\sigma=0
$$

is expressed in terms of the stress invariants $I_{1}$ and $J_{2}$ of the Kirchhoff stress tensor and the damage threshold $\sigma$ represents the material toughness to micro-defect propagation. In Eq. (6) the variables $\alpha$ and $\beta$ represent damage mode parameters corresponding to the different damage mechanisms acting on the micro-level: shear modes for negative stress triaxialities, void-growth-dominated modes for large positive triaxialities and mixed modes (simultaneous growth of voids and evolution of micro-shear-cracks) for lower positive stress triaxialities. In addition, the influence of the Lode parameter is taken into account because it has been shown that its effect on the evolution of the microstructure is remarkable especially in low stress triaxiality regions. Therefore, the damage mode parameters $\alpha$ and $\beta$ in Eq. (6) depend on the stress intensity $\sigma_{e q}=\sqrt{3 J_{2}}$ (von Mises equivalent stress), the stress triaxiality

$$
\eta=\frac{\sigma_{m}}{\sigma_{e q}}=\frac{I_{1}}{3 \sqrt{3 J_{2}}}
$$

defined as the ratio of the mean stress $\sigma_{m}$ and the von Mises equivalent stress $\sigma_{e q}$ as well as on the Lode parameter

$$
\omega=\frac{2 T_{2}-T_{1}-T_{3}}{T_{1}-T_{3}} \quad \text { with } T_{1} \geq T_{2} \geq T_{3}
$$

expressed in terms of the principal stress components $T_{1}$, $T_{2}$ and $T_{3}$.

For the tested aluminum alloy the dependence of $\alpha$ and $\beta$ on stress state has been examined in detail performing numerical simulations on the micro-scale by [4]. For practical applications simplified functions have been proposed by [14] still allowing accurate modeling of failure behavior observed in different experiments. It is worthy to note that the parameters $\alpha$ and $\beta$ correspond to different damage and fracture mechanisms acting on the micro-level. Therefore, they can be seen to be typical parameters for a wide class of metals and not only for the aluminum alloy under investigation. Thus, only the damage threshold $\sigma$ in the damage criterion (6) is a material parameter whereas $\alpha$ and $\beta$ are not.

In particular, the parameter $\alpha$ is taken to be

$$
\alpha(\eta)=\left\{\begin{array}{l}
-0.15 \text { for } \eta \leq 0 \\
0.33 \text { for } \eta>0
\end{array}\right.
$$

and the parameter $\beta$ is given by the non-negative function

$$
\beta(\eta, \omega)=\beta_{0}(\eta, \omega=0)+\beta_{\omega}(\omega) \geq 0,
$$

with

$$
\beta_{0}(\eta)=-1.28 \eta+0.85
$$

and

$$
\beta_{\omega}(\omega)=-0.017 \omega^{3}-0.065 \omega^{2}-0.078 \omega .
$$

Furthermore, the damage strain rate tensor can be determined by the damage rule

$$
\dot{\mathbf{H}}^{d a}=\dot{\mu}\left(\bar{\alpha} \frac{1}{\sqrt{3}} \mathbf{1}+\bar{\beta} \mathbf{N}\right)
$$

where $\dot{\mu}$ is a non-negative scalar-valued factor. In Eq. (13) the normalized stress related deviatoric tensor $\mathbf{N}=$ $\frac{1}{2 \sqrt{J_{2}}} \operatorname{dev} \tilde{\mathbf{T}}$ has been used and $\dot{\mu}$ represents in the proposed continuum damage approach the equivalent damage strain rate measure characterizing the amount of increase in irreversible damage. The parameters $\bar{\alpha}$ and $\bar{\beta}$ are kinematic variables describing the portion of volumetric and isochoric damage-based deformations. These parameters also correspond to different damage and fracture mechanisms on the micro-scale. Again, they are based on numerical analyses with void-containing unit cells under various three-dimensional loading conditions [4] as well as 
on comparison of test results and numerical simulations of different experiments with uniaxially and biaxially loaded specimens [14].

In particular, the parameter $\bar{\alpha}$ characterizing the amount of volumetric damage strain rates caused by isotropic volume change of micro-defects is given by

$$
\bar{\alpha}(\eta)=\left\{\begin{array}{l}
0 \text { for } \eta \leq 0 \\
0.5714 \eta \text { for } 0<\eta \leq 1.75 \\
1 \text { for } \eta>1.75
\end{array} .\right.
$$

The parameter $\bar{\beta}$ corresponding to the amount of anisotropic isochoric damage strain rates caused by evolution of micro-shear-cracks is taken to be

$$
\bar{\beta}(\eta, \omega)=\bar{\beta}_{0}(\eta)+f_{\beta}(\eta) \bar{\beta}_{\omega}(\omega)
$$

with

$$
\begin{gathered}
\bar{\beta}_{0}(\eta)=\left\{\begin{array}{l}
0.87 \text { for } \eta \leq \frac{1}{3} \\
0.979-0.326 \eta \text { for } \frac{1}{3}<\eta \leq 3 \\
0 \text { for } \eta>3
\end{array}\right. \\
f_{\beta}(\eta)=-0.0252+0.0378 \eta
\end{gathered}
$$

and

$$
\bar{\beta}_{\omega}(\omega)=\left\{\begin{array}{l}
1-\omega^{2} \text { for } \eta \leq \frac{2}{3} \\
0 \text { for } \eta>\frac{2}{3}
\end{array} .\right.
$$

It can be summarized that the macroscopic damage rule (13) takes into account volumetric parts (first term in Eq. (13)) corresponding to isotropic growth of voids on the micro-scale as well as deviatoric parts (second term in Eq. (13)) corresponding to anisotropic evolution of microshear-cracks, respectively. Therefore, both basic damage mechanisms discussed above (growth of isotropic voids and evolution of micro-shear-cracks) acting on the microlevel are involved in the macroscopic damage rule (13) of the phenomenological continuum model. In addition, it has been shown in [20] that the shear deformation component activated damage mechanisms in the small negative stress triaxiality regime. In numerical simulations this behavior can be taken into account by the deviatoric parts in Eq. (13) in a phenomenological manner.

\section{Experiments and numerical simulations}

The investigated material is an aluminum alloy of series 2017. The phenomenological continuum model discussed above takes into account different material parameters which have to be identified because they are needed as input data in numerical calculations. Determination of constitutive parameters is based on fitting of experimental curves of uniaxial tests and has been discussed in detail by [14]. Briefly, elastic behavior is modeled with Young's

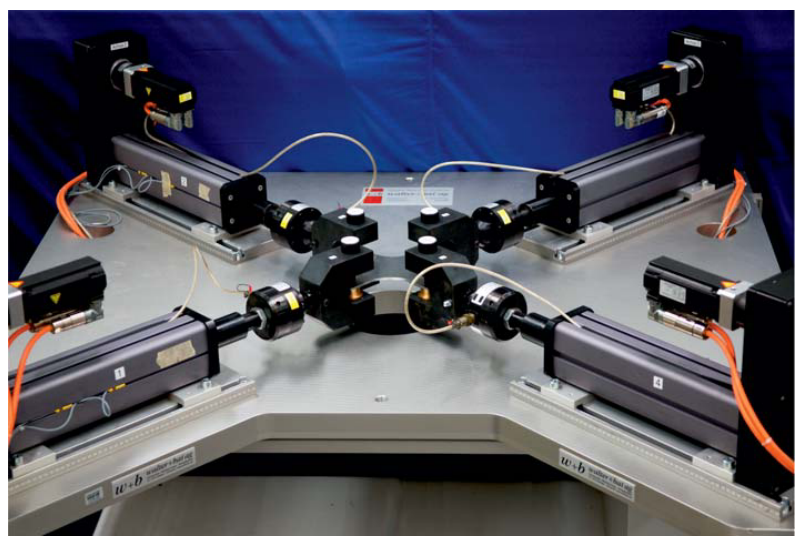

Figure 1. Biaxial testing machine

modulus $E=65,000 \mathrm{MPa}$ and Poisson's ratio $v=0.3$. Plastic behavior is governed by the hardening law

$$
c=c_{0}\left(\frac{H \gamma}{n c_{0}}+1\right)^{n}
$$

with the initial yield strength $c_{0}=174 \mathrm{MPa}$, the hardening modulus $H=1800 \mathrm{MPa}$ and the hardening exponent $n=0.23$. Onset of damage is characterized by the damage threshold $\sigma=295 \mathrm{MPa}$ whereas the effect of damage on elastic behavior is simulated by the parameters $\eta_{1}=-100,000 \mathrm{MPa}, \eta_{2}=-50,000 \mathrm{MPa}$, $\eta_{3}=-12,500 \mathrm{MPa}$, and $\eta_{4}=-2500 \mathrm{MPa}$.

Many metal forming processes are carried out under compressive loading conditions and, therefore, there is remarkable demand for experiments to examine in detail inelastic deformation behavior as well as damage and failure in the regime of negative stress triaxialities. A new experimental program has been developed to propose new tests revealing the effect of stress state on inelastic behavior, damage and fracture in ductile metals undergoing combined tensile, shear and compressive loading conditions [13]. The experiments are performed using the biaxial testing machine type LFM-BIAX $20 \mathrm{kN}$ (produced by Walter \& Bai, Switzerland) shown in Fig. 1. It contains four electro-mechanically, individually driven cylinders with load maxima and minima of $\pm 20 \mathrm{kN}$ (tension and compression loading are possible). The respective specimens are fixed in the four heads of the cylinders where clamped or hinged boundary conditions are possible.

Specimens with special geometry are biaxially loaded leading to shear-tension and shear-compression mechanisms in their central parts where localized inelastic deformations and final failure occur. The experimental equipment discussed above and this geometry of specimens are used here to study the deformation and failure behavior in the range of negative stress triaxialities. In addition, corresponding numerical calculations deliver detailed information on distributions and amounts of stress and strain measures especially in critical regions of the specimens. The numerical analyses are carried out using the finite element program ANSYS enhanced by a user-defined ma- 


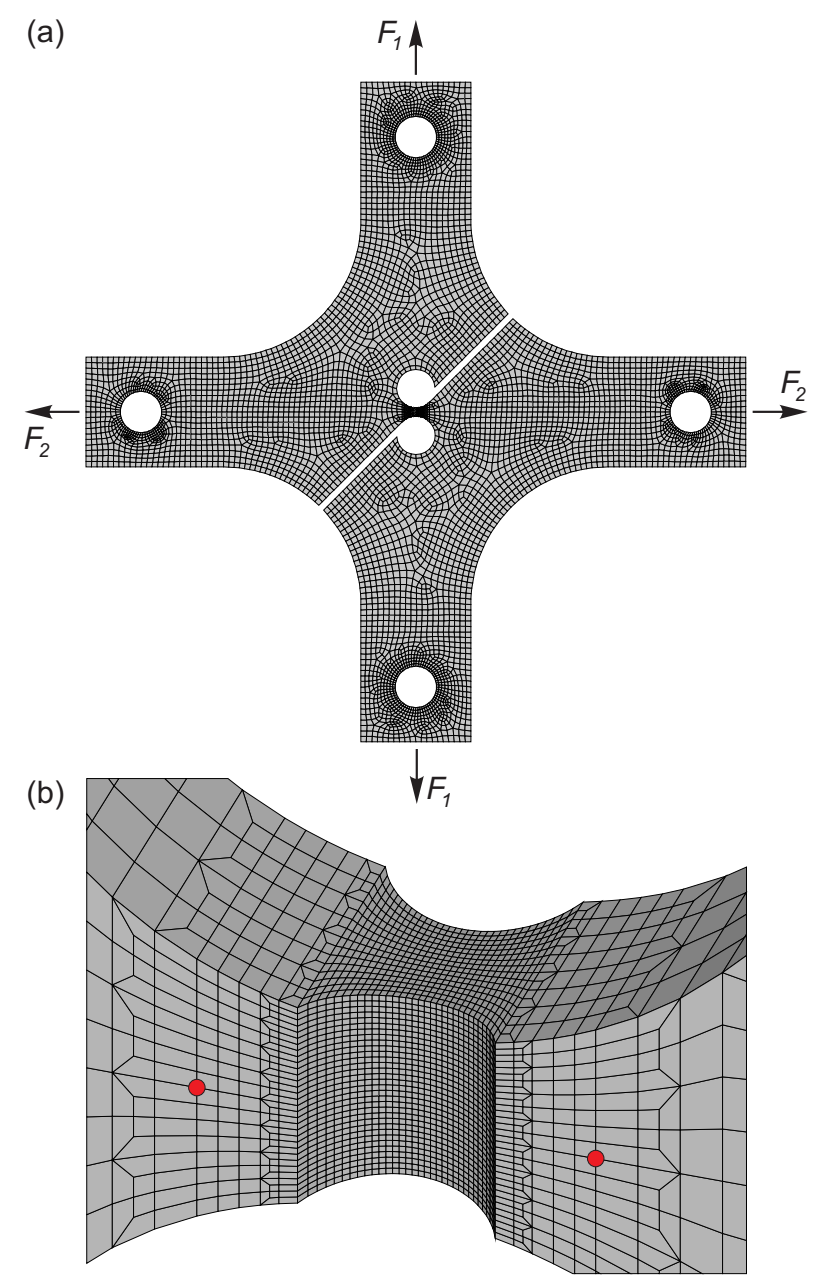

Figure 2. (a) Specimen, loading conditions and finite element mesh, (b) Detail of the notched central part

terial subroutine based on the continuum model discussed above.

Geometry, finite element mesh and loading conditions of the flat specimen are shown in Fig. 2(a). The numerical calculations are based on finite element meshes with 42,248 eight-node-elements of type Solid185. During the tests the specimens are simultaneously loaded in vertical and horizontal direction by the forces $F_{1}$ and $F_{2}$. The vertical load $F_{1}$ leads to shear mechanisms in the center of the specimen whereas the load $F_{2}$ leads in this part to superimposed tension or compression modes. In the present paper, results of experiments and of corresponding numerical simulations for the load ratio $F_{1}: F_{2}=1:-1.5$ will be discussed in detail which are seen to be relevant for metal forming processes. In the center of the specimen additional notches in thickness direction have been milled, see Fig. 2(b), leading to localization of inelastic deformations, damage and final fracture in this part. Here, refinement of the finite element mesh has been used to accurately cover high gradients of stress and strain quantities. This part of the specimen is $3 \mathrm{~mm}$ high with thickness of $2 \mathrm{~mm}$ and the radius of the notch is $2 \mathrm{~mm}$. Displacement measures analyzed and discussed in the present pa-

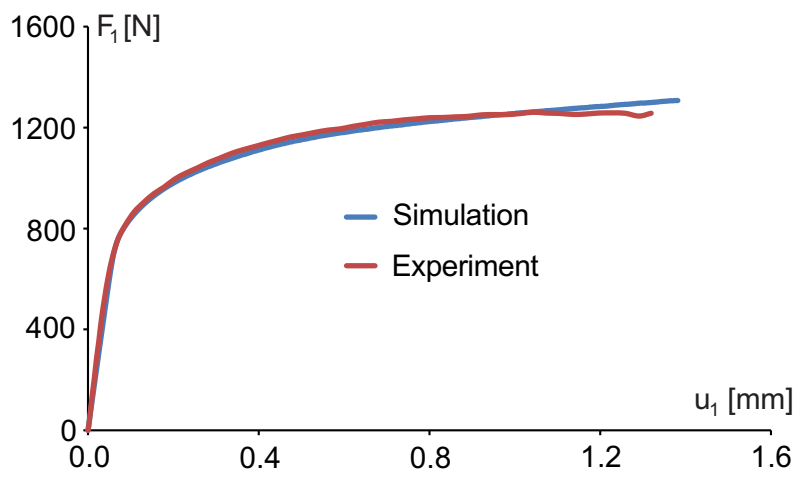

Figure 3. Load-displacement curves $F_{1}-u_{1}$

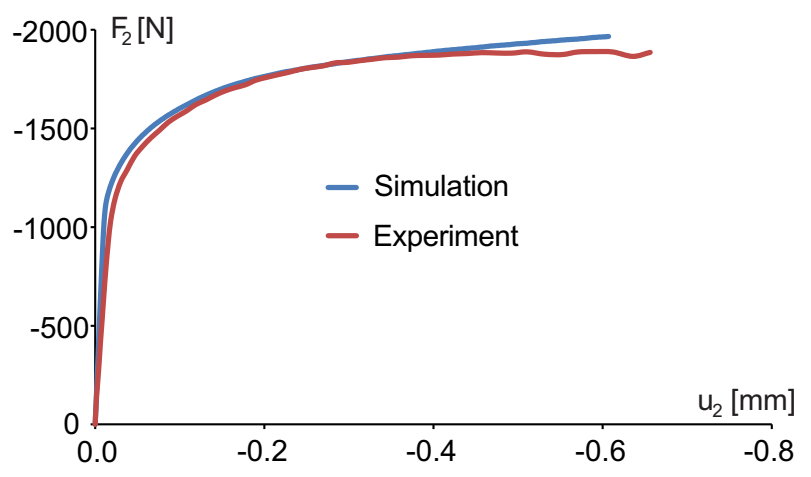

Figure 4. Load-displacement curves $F_{2}-u_{2}$

per correspond to movements of the red points shown in Fig. 2(b). For example, the vertical displacement $u_{1}$ is the vertical relative movement of these points whereas the displacement $u_{2}$ is the horizontal one. During the experiments, these displacement are recorded by digital image correlation discussed below and in the numerical simulations displacements of corresponding finite element nodes are computed.

Results of experiments and of corresponding numerical simulations are analyzed and compared to elucidate localized deformation and failure behavior. In particular, load-displacement curves $F_{1}-u_{1}$ are shown in Fig. 3. After elastic loading a large region with inelastic behavior including hardening occurs. The load maximum is about $F_{1}=1300 N$. Final fracture of the specimen occurred at $u_{1}=1.3 \mathrm{~mm}$ but no remarkable softening behavior can be observed before this point has been reached. The numerically predicted curve based on the continuum model discussed above shows very good agreement with the experimental one. In addition, the load-displacement curves for the other direction, $F_{2}-u_{2}$, are shown in Fig. 4 with load minimum of $F_{2}=-1900 \mathrm{~N}$ and the final displacement $u_{2}=-0.65 \mathrm{~mm}$. Again, very good agreement of experimental and numerically predicted curves can be observed.

In the load-displacement curves (Figs. 3 and 4) small waves can be observed especially shortly before final fracture occurred. This behavior is typical for shearcompression loading conditions and has also been observed for other negative stress ratios, see for example 


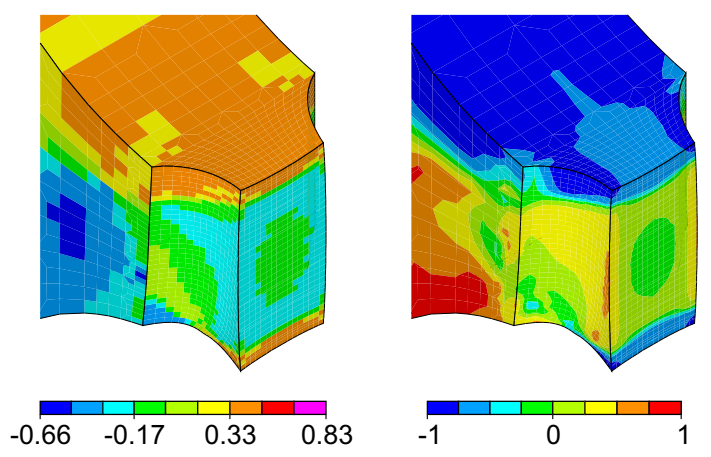

Figure 5. Stress triaxiality $\eta$ and Lode parameter $\omega$

[14]. One reason for this behavior may be evolution of localized inelastic shear deformations caused by formation of shear-cracks. Due to superimposed compression loading continuation of these shear deformations stops because crack areas come into contact and are pressed together. It is worthy to note that this behavior does not mean healing of the locally failed material because the shear-cracks still exist and will be again active when associated shear forces increase during further loading of the specimen.

The load-displacement curves discussed above only demonstrate the global behavior of the specimen for the chosen loading condition. Therefore, further analyses are recommended to study the local damage and failure behavior especially in critical regions where inelastic deformations are localized and final fracture will occur. For example, numerically predicted distributions of the stress triaxiality $\eta$ (Eq. 7) and the Lode parameter $\omega$ (Eq. 8) at the end of the loading process shortly before final fracture occurs will be discussed. These stress parameters in a vertical cut in the central region of the specimen are shown in Fig. 5. In particular, for the considered load ratio $F_{1}: F_{2}=1:-1.5$ the stress triaxiality is here nearly constant in the central area with $\eta=-0.2$ and only in the upper and lower boundaries positive stress triaxialities of about $\eta=0.33$ occur caused by the tensile deformation of these parts caused by the shear loading. In addition, similar stress parameter distribution can be seen for the Lode parameter. It is about $\omega=0.0$ in the center and reaches $\omega=-0.7$ at the boundaries. For these values in the central area of the specimen damage and failure will be caused by formation and growth of micro-shear cracks whereas the effect of nucleation and growth of micro-voids will be marginal which is typical for shear-compression loading conditions. Combination of voids and shear-cracks on the micro-level will only occur in the boundaries of the notched region.

The amount of damage strains can be quantified by the equivalent damage strain measure $\mu$, see Eq. (13). Figure 6 shows its numerically predicted distribution in the notched part of the specimen at the end of the numerical simulation, shortly before fracture occurred in the experiments. The equivalent damage strain $\mu$ shows its maxima of about $\mu=4 \%$ at the boundaries of the notch but also about $\mu=2 \%$ in the band between these points. Since

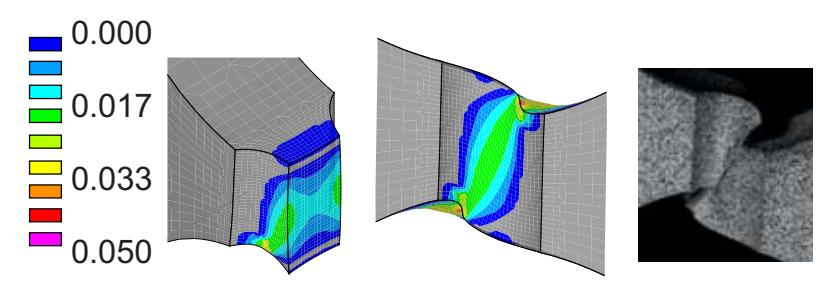

Figure 6. Equivalent damage strain $\mu$ and experimental fracture line

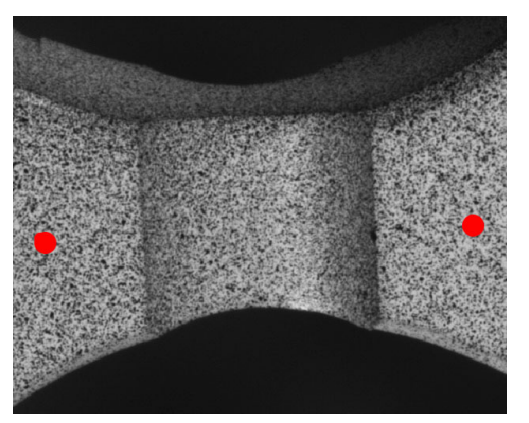

Figure 7. Speckle pattern of the specimen's center incl. emphasis of points (red) for displacement measurement

these values of the equivalent damage strain are predicted to only occur in this band, the onset of macro-cracking will be in this part and the crack then runs along the band of moderate equivalent damage strains which are the weakest points of the damaged material. This numerically predicted band nicely corresponds to the fracture line of the tested specimen also shown in Fig. 6. In addition, the numerically predicted geometry of the inelastically deformed central part of the specimen nicely corresponds to the geometry of the tested specimen shown in the photo.

Digital image correlation (DIC) technique has been applied in the present experimental program to analyze deformation behavior in the critical zones of the specimens. During the shear-compression test the three-dimensional displacement field of the specimen surface was measured with a Q-400 digital 3D image correlation system provided by Dantec/Limess. The stereo setting consists of two 2.0Mpx cameras equipped with Ricoh FL-CC7528$2 \mathrm{M} 75 \mathrm{~mm} \mathrm{~F} / 2.8$ lenses and extension tubes of $10 \mathrm{~mm}$. The evaluation was realized with the corresponding Istra 4D software provided with the system. Specimen preparation was realized shortly before the experiment started. One side of the specimen was firstly sprayed with a white acrylic lacquer and then the speckle pattern shown in Fig. 7 was sprayed on with a graphite-based coating. It is worthy to note that no ex-foliation of the coating was observed during the experiment. The displacements of the selected points marked in red in Fig. 7 as well as the forces in axes directions were transferred and stored with the data sets of the image correlation system at a frequency of $0.25 \mathrm{~Hz}$ while the velocity of the machine was $0.04 \mathrm{~mm} / \mathrm{min}$.

Strain fields of the tested specimens analyzed by DIC in the load steps shortly before final fracture occurs are 
(a)
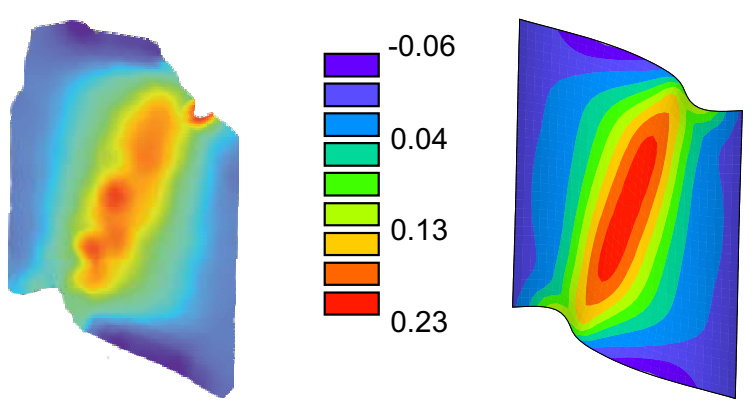

(b)
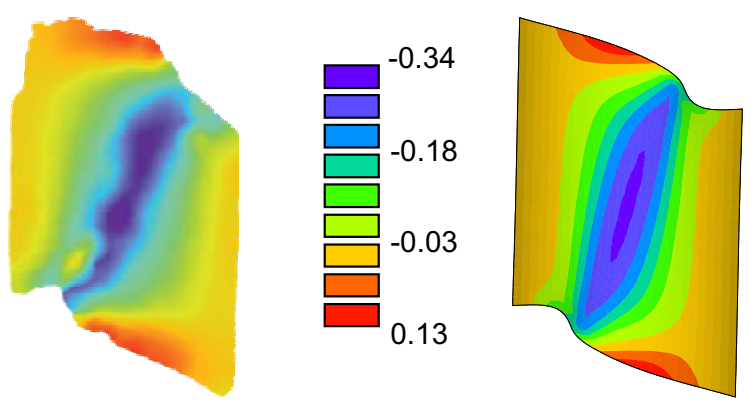

(c)
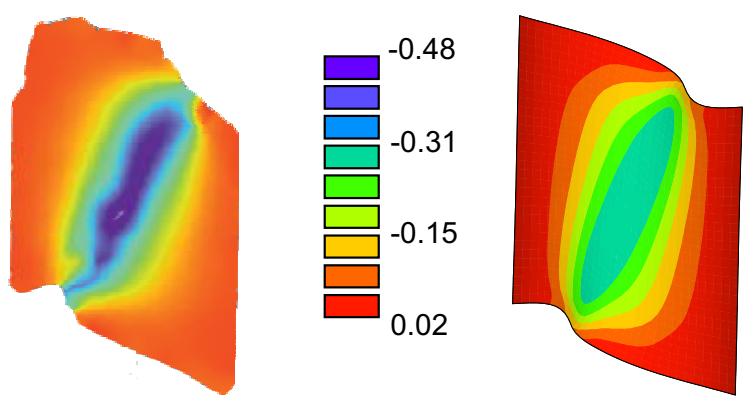

Figure 8. Strain fields taken from DIC (left) and numerical simulation (right): (a) Normal strain in direction 1, (b) Normal strain in direction 2, (c) Shear strain

compared with corresponding fields predicted by numerical simulations based on the continuum model discussed above. Since the theoretical approach takes into account elastic, plastic and damage strain rate tensors the comparison of measured and numerically predicted strain fields can also be seen as validation of the flow rule (4) and the damage rule (13). To visualize numerically obtained strain fields at the boundaries of the specimen's center thin shell elements with marginal stiffness have been overlayed and numerically predicted strain measures are taken from these elements. The experimentally measured strains shown in Fig. 8 are represented with respect to a coordinate system with origin at the center of the notch where the $z$-axis is defined perpendicular to the specimen surface and a further axis-direction is defined in tensile direction (Fig. 2) of the current step while the predicted numerical results are represented with respect to the global coordinate system. Thus, both coordinate systems coincide quite well, which is not easy to achieve on the experimental side.
Figure 8(a) shows the distribution of the normal strain component in direction 1 (see Fig. 2) whereas the normal strain component in direction 2 is visualized in Fig. 8(b) and the shear strain in Fig. 8(c). In particular, the normal strain component in direction 1 analyzed by DIC (left picture in Fig. 8(a)) is localized in a small band with maximum values of $23 \%$. This distribution and these values are also obtained from the corresponding numerical simulation (right picture in Fig. 8(a)). In addition, the distributions of the normal strain component in direction 2 analyzed by DIC and that one obtained by the numerical simulation are visualized in Fig. 8(b). A similar localized band of this normal strain component can be seen with minimum values of $-34 \%$ caused by the superimposed compression loading. Again, for these experimental and numerically predicted normal strains, distributions and values agree very well. Furthermore, distributions of the shear strains is shown in Fig. 8(c). Experimental shear strains analyzed by DIC (left picture in Fig. 8(c)) are also localized in a small band with values up to $48 \%$. In comparison, numerically predicted shear strains (right picture in Fig. 8(c)) show similar distribution but the highest values are only about $30 \%$. One reason for this quantitative difference is the possible occurrence of larger shearcracks in this band. Their effect has also been observed in the load-displacement curves as mentioned above (small waves shortly before final fracture occurs, see Figs. 3 and 4). These shear-cracks lead to further softening of the damaged material and, thus, to larger shear strains in this localized band but due to remarkable superimposed compression and associated contact of the crack areas this will not lead to subsequent final fracture. However, this formation of larger shear-cracks cannot be modeled by the proposed continuum model discussed above because such discontinuities cannot be taken into account. It is worthy to note that this discrepancy only in quantities of experimental and numerically predicted shear strains has also be observed for other load ratios in the shear-compression range [14] and will be analyzed in more detail by the authors in near future.

\section{Conclusions}

The paper has discussed an anisotropic continuum damage and failure model for ductile metals. The phenomenological approach is based on kinematic definition of damage tensors and takes into account the effect of stress state on damage conditions and damage strain evolution laws. Different branches of these criteria based on experimental studies and numerical simulations are considered corresponding to various microscopic damage and fracture mechanisms depending on stress intensity, stress triaxiality and the Lode parameter. Damage mode functions for a wide class of metals have been proposed which are able to simulate all relevant effects observed in different experiments and will allow practical applicability.

Experiments with biaxially loaded specimens undergoing shear-compression loading appearing in metal forming processes have been performed. Digital image corre- 
lation technique has been used to analyze current strain fields in critical regions of the specimens.

Numerical simulations corresponding to the experiments have been performed. Detailed information on loaddeformation behavior as well as on stress and strain states have been analyzed and compared with available experimental data especially in the critical regions of the specimens where large inelastic deformations and ductile failure occurred. The results of the presented experimentalnumerical procedure allow validation of the proposed stress-state-dependent constitutive equations for moderate negative stress triaxialities. It seems that under shearcompression loading conditions cracks occur in the center of the specimen but their areas come into contact and are pressed together. Thus, the activity of these cracks will be interrupted for a while, but they are still existent. This failure may play an important role for later use of formed products and might lead to remarkable reduction in their safety and lifetime. This effect will be analyzed in more detail by the authors in near future.

In summary, the proposed constitutive model can be used in numerical calculations to optimize sheet and bulk metal forming processes. In addition, it has been shown that the final macro-cracks are initiated in points of moderate equivalent damage strains and following fracture lines of the respective failed specimens occur in the band of maximum equivalent damage strains which might give reference to formulation of alternative engineering fracture criteria based on damage strain measures.

\section{References}

[1] M. Kuna, D. Sun, Int. J. Fract. 81, 235-258 (1996)

[2] X. Gao, J. Kim, Int. J. Sol. Struct. 43, 6277-6293 (2006)
[3] I. Barsoum, J. Faleskog, Int. J. Sol. Struct. 48, 925-938 (2011)

[4] M. Brünig, S. Gerke, V. Hagenbrock, Int. J. Plast. 50, 49-65 (2013)

[5] M. Brünig, S. Gerke, V. Hagenbrock, Int. J. Plast. 63, 49-63 (2014)

[6] Y. Bai, T. Wierzbicki, Int. J. Mech. Sci. 46, 81-98 (2004)

[7] X. Gao, G. Zhang, C. Roe, Int. J. Damage Mech. 19, 75-94 (2010)

[8] M. Dunand, D. Mohr, J. Mech. Phys. Sol. 59, 13741394 (2011)

[9] M. Brünig, O. Chyra, D. Albrecht, L. Driemeier, M. Alves, Int. J. Plast. 24, 1731-1755 (2008)

[10] L. Driemeier, M. Brünig, G. Micheli, M. Alves, Mech. Mat. 42, 207-217 (2010)

[11] Y. Bai, T. Wierzbicki, Int. J. Plast. 24, 1071-1096 (2008)

[12] T. Kuwabara, Int. J. Plast. 23, 385-419 (2007)

[13] M. Brünig, D. Brenner, S. Gerke, Eng. Fract. Mech. 141, 152-169 (2015)

[14] M. Brünig, S. Gerke, M. Schmidt, Int. J. Fract. (accepted for publication) (2016)

[15] M. Brünig, Int. J. Plast. 19, 1679-1713 (2003)

[16] W.A. Spitzig, R.J. Sober, O. Richmond, Metall. Trans. 7A, 1703-1710 (1976)

[17] M. Brünig, Int. J. Plast. 15, 1237-1264 (1999)

[18] H. Badreddine, K. Saanouni, T.D. Nguyen, Int. J. Sol. Struct. 63, 11-31 (2015)

[19] C. Chow, J. Wang, Eng. Fract. Mech. 27, 547-558 (1987)

[20] S. Kweon, Eur. J. Mech. A/Solids 31, 203-212 (2012) 